\title{
An Open Problem in Complex Analytic Geometry Arising in Harmonic Analysis
}

\author{
M. Ruzhansky * \\ Department of Mathematics, Imperial College London \\ 180 Queen's Gate, London SW7 2AZ, United Kingdom
}

\begin{abstract}
In this paper, an open problem in the multidimensional complex analysis is presented that arises in the harmonic analysis related to the investigation of the regularity properties of Fourier integral operators and in the regularity theory for hyperbolic partial differential equations. The problem is discussed in a self-contained elementary way and some results towards its resolution are presented. A conjecture concerning the structure of appearing affine fibrations is formulated.
\end{abstract}

Keywords and phrases: complex analytic geometry, fibrations, Lagranigian manifolds, Fourier integral operators

Mathematics Subject Classification: 35S30, 58J40, 32A30, 53D12

\section{Introduction}

In this paper, we present an open problem that arises in the harmonic analysis related to Fourier integral operators and hyperbolic partial differential equations. In [10], Seeger, Sogge, and Stein formulated a so-called smooth factorization condition that is related to the local $L^{p}$ bounds for non-degenerate Fourier integral operators. In [7] it was shown that this condition is satisfied in a number of important cases from the point of view of the theory of hyperbolic partial differential equations. Moreover, an approach to this condition based on the notion of so-called affine fibrations has been developed in [7]. In [9], regularity properties of Fourier integral operators for certain parameter dependent affine fibrations have been established.

The problem is of independent interest since it describes a fibration structure in Lagrangian manifolds. Recently, this condition reappeared in [3] as a condition on the space variables in the estimates for Fourier integral operators on Fourier Lebesgue spaces.

In this paper we will reformulate this condition entirely in the language of complex analytic geometry of several complex variables. Subsequently, we will present results that have been established for this problem by the author. This analysis together with available examples suggests that the structure of the set of singularities of affine fibrations must have a rather rigid nature. In this paper we will formulate the corresponding conjecture that is motivated by results and examples presented here. Combined with other

*Corresponding author. E-mail: m.ruzhansky@imperial.ac.uk 
methods developed in [7] and [9], the validity of this conjecture would provide an approach to the sharp Sobolev $L^{p}$ properties of the corresponding non-degenerate Fourier integral operators and of solutions to the Cauchy problems for corresponding hyperbolic partial differential equations. Thus, an additional purpose of this paper is to attract the attention of complex analysts to a problem of importance for symplectic geometry and for Fourier integral operators.

In the following section we formulate the problem, present some results and examples that motivate a conjecture about the set of essential singularities of the corresponding set of affine fibrations. In the last section we will explain how this problem arises naturally in the symplectic geometry and the subsequent theory of Fourier integral operators.

\section{Formulation of the problem}

In this section we will formulate the problem in question in a simple self-contained way in the language of the complex analysis of several variables.

Let $\Omega$ be an open connected subset of $\mathbb{C}^{n}$, and let $\Gamma: \Omega \rightarrow \mathbb{C}^{n}$ be a holomorphic mapping (in general, the image space does not have to have the same dimension as $\Omega$, but we consider this case for simplicity). Let $D \Gamma(\xi) \in \mathbb{C}^{n \times n}$ denote the Jacobi matrix of first order derivatives of $\Gamma$, so that its entries are $\{D \Gamma(\xi)\}_{j k}=\partial_{\xi_{k}} \Gamma_{j}(\xi), j, k=1, \ldots, n$, where we denote $\Gamma=\left(\Gamma_{1}, \ldots, \Gamma_{n}\right)$.

Let us make the following assumptions about the mapping $\Gamma$ :

(A1) Let us denote

$$
k:=\max _{\xi \in \Omega} \operatorname{rank} D \Gamma(\xi)
$$

and assume that $1 \leq k \leq n-1$. Let $\Omega^{(k)}$ denote the set of $\xi$ where the rank of $D \Gamma$ is maximal, i.e.

$$
\Omega^{(k)}=\{\xi \in \Omega: \operatorname{rank} D \Gamma(\xi)=k\} .
$$

(A2) Assume that for every $\xi \in \Omega^{(k)}$, the level set

$$
\Gamma^{-1}(\Gamma(\xi))=\{\eta \in \Omega: \Gamma(\eta)=\Gamma(\xi)\}
$$

is an affine $(n-k)$-dimensional space through $\xi$.

Let us now discuss these conditions. In fact, condition (A1) is not restrictive since it excludes the cases $k=0$ and $k=n$ only, where the subsequent problem is trivial. The case of $k=0$ would mean that the rank of $D \Gamma$ is identically zero, which would mean that $\Gamma$ is a constant mapping. The case of $k=n$ would mean that the level sets of $\Gamma$ are just points. Since we will be interested in the geometry of the level sets of $\Gamma$, we exclude these two cases from the consideration.

Condition (A2) is the main condition that we impose on $\Gamma$. In fact, for $\xi \in \Omega^{(k)}$, by the implicit function theorem we may conclude that the level set $\Gamma^{-1}(\Gamma(\xi))$ is a smooth analytic $(n-k)$-dimensional submanifold of $\Omega$. Condition (A2) assumes that all these level sets are affine (i.e. they are linear spaces with the origin at $\xi$ ), thus imposing a rigid structure on the geometry of the problem.

We can also note that the level sets $\Gamma^{-1}(\Gamma(\xi))$ can not intersect in $\Omega^{(k)}$ (unless they coincide). Indeed, if they would intersect, the intersection point would belong to two level sets. Thus, the mapping $\Gamma$ would take the same value on both level sets. Since both of them are linear by (A2), and if the intersection point is in $\Omega^{(k)}$, the joint level set can be regular only if they coincide. In this way the set $\Omega^{(k)}$ becomes a union of non-intersecting affine spaces which are the level sets of $\Gamma$. So, we can write

$$
\Omega^{(k)}=\bigcup_{\xi \in \Omega^{(k)}}\left(\Gamma^{-1}(\Gamma(\xi)) \cap \Omega^{(k)}\right),
$$

where any two affine spaces $\Gamma^{-1}(\Gamma(\xi))$ from the union are either disjoint in $\Omega^{(k)}$ or coincide. Thus, we will talk about an affine fibration in $\Omega^{(k)}$, given by the union of disjoint planes (which are level sets of a holomorphic mapping $\Gamma$ ). 
It follows from (A1) that the set $\Omega^{(k)}$ is open and dense in $\Omega$. Let us now denote

$$
\varkappa: \xi \mapsto \operatorname{ker} D \Gamma(\xi), \quad \varkappa: \Omega^{(k)} \rightarrow \mathbb{G}_{n-k}\left(\mathbb{C}^{n}\right),
$$

so that by the implicit function theorem $\varkappa$ is a regular mapping from $\Omega^{(k)}$ to the Grassmanian $\mathbb{G}_{n-k}\left(\mathbb{C}^{n}\right)$, which is the set of all $(n-k)$-dimensional linear subspaces of $\mathbb{C}^{n}$. We note that condition (A2) implies that for $\xi \in \Omega^{(k)}$ we have

$$
\Gamma^{-1}(\Gamma(\xi))=\xi+\varkappa(\xi),
$$

so that the dependence of the level sets on $\xi \in \Omega^{(k)}$ is analytic.

The main question of this paper is when (and whether) this fibration extends analytically from $\Omega^{(k)}$ to the set $\Omega$. In fact, it can be shown that the mapping $\varkappa$ extends to a meromorphic mapping in $\Omega$, so by the analytic graph theorem this extension is analytic if and only if it is continuous (see e.g. [2]). We denote this extension by $\bar{\varkappa}$.

To study the extension properties of $\varkappa$ from $\Omega^{(k)}$ to $\Omega$, let us introduce the set

$$
\Omega^{\text {sing }}=\{\xi \in \Omega: \varkappa \text { is not continuously extendible over } \xi\},
$$

which is the set of essential singularities of $\bar{\varkappa}$. The condition that $\varkappa$ extends regularly from $\Omega^{(k)}$ to $\Omega$ would be equivalent to the condition that the set $\Omega^{\text {sing }}$ is empty.

Now we will present some results, examples, and formulate a conjecture concerning the structure of the set $\Omega^{\text {sing }}$ for the mappings $\Gamma$ satisfying conditions (A1) and (A2). The following theorems have been established in [5], and then extended in [7]. Thus, it can be shown that the mapping

$$
\bar{\varkappa}: \Omega \rightarrow \mathbb{G}_{n-k}\left(\mathbb{C}^{n}\right)
$$

is meromorphic in the sense of [4] (see also [2]). There, a mapping $\tau: X \rightarrow Y$ between complex manifolds $X$ and $Y$ is called meromorphic if the following three conditions hold:

(1) For every $x \in X$ the image set $\tau(x) \subset Y$ is non-empty and compact in $Y$.

(2) The graph of the mapping $\tau$, that is the set all pairs $(x, y) \in X \times Y$ such that $y \in \tau(x)$, is a connected complex analytic subset of $X \times Y$ of dimension equal to the dimension of $X$.

(3) There exist a dense subset $X^{*}$ of $X$, such that for every $x \in X^{*}$ the image set $\tau(x)$ consists of a single point.

This immediately implies that the set $\Omega^{\text {sing }}$ of essential singularities of a meromorphic mapping $\bar{\varkappa}$ is analytic and we have the estimate $\operatorname{dim} \Omega^{\text {sing }} \leq n-2$. With additional analysis, we have more:

Theorem 2.1. Suppose $\Omega^{\text {sing }} \neq \emptyset$. Then $\Omega^{\text {sing }}$ is analytic and for every $\xi \in \Omega$ we have the estimate

$$
\max \{k-1, n-k+1\} \leq \operatorname{dim}_{\xi} \Omega^{\text {sing }} \leq n-2 .
$$

Moreover, let $\xi \in \Omega^{\mathrm{sing}}$ be a regular point of the analytic set $\Omega^{\mathrm{sing}}$. Let $\xi=\lim _{j \rightarrow \infty} \xi_{j}$ be a limit of some sequence $\xi_{j} \in \Omega^{(k)}$, and let $\varkappa\left(\xi_{j}\right) \rightarrow \varkappa \in \mathbb{G}_{n-k}\left(\mathbb{C}^{n}\right)$. Then we have the inclusion $\varkappa \subset T_{\xi} \Omega^{\text {sing. }}$.

We note that a sequence $\varkappa\left(\xi_{j}\right)$ always has a convergent subsequence since the Grassmanian is compact, so we can start with a sequence $\xi_{j} \in \Omega^{\text {sing }}$ in Theorem 2.1 for which the corresponding sequence $\varkappa\left(\xi_{j}\right)$ converges to some $\varkappa \in \mathbb{G}_{n-k}\left(\mathbb{C}^{n}\right)$. As a corollary of estimates of Theorem 2.1, we obtain that affine fibrations always have regular extensions from $\Omega^{(k)}$ to $\Omega$ in lower dimensions:

Corollary 2.2. We have the following statements:

(1) if $n \leq 3$, then $\Omega^{\text {sing }}$ is empty.

(2) if $k \leq 2$, then $\Omega^{\text {sing }}$ is empty.

We can also show that the estimates on the dimension in Theorem 2.1 are sharp: 
Theorem 2.3 (Sharpness). Let $k$ and d satisfy

$$
3 \leq k \leq n-1 \text { and } \max \{k-1, n-k+1\} \leq d \leq n-2 .
$$

Then there exists a holomorphic mapping $\Gamma: \mathbb{C}^{n} \rightarrow \mathbb{C}^{n}$ satisfying conditions (A1), (A2) with $k$ as above, such that $\operatorname{dim} \Omega^{\text {sing }}=d$.

Corollary 2.2 shows that the smallest dimension for which a singular fibration can exist is $n=4$, with $k=3$. Let us show that in $\mathbb{C}^{4}$ there is a fibration by lines, which also satisfies additional property

$$
\Gamma=\nabla \psi
$$

for some holomorphic function $\psi: \Omega \rightarrow \mathbb{C}$. We may call the corresponding fibrations the fibrations of gradient type. In fact, in the following section we will show how such fibrations naturally arise in the symplectic geometry of Lagrangian manifolds and related Fourier integral operators, in which cases condition (2.1) is satisfied with a function $\psi$ obtained from function $\phi$ from the following section by factoring out the homogeneous direction. Under (2.1) we obviously have $D \Gamma=D^{2} \psi$, which is a symmetric matrix.

Now we will give some examples of mappings in Theorem 2.3. Define

$$
\psi\left(\xi_{1}, \xi_{2}, \xi_{3}, \xi_{4}\right)=\xi_{1} \xi_{2}^{2}+\left(\xi_{3}-\xi_{2} \xi_{4}\right)^{2} .
$$

The Hessian of the function $\psi$ has the form

$$
D^{2} \psi(\xi)=\left(\begin{array}{cccc}
0 & 2 \xi_{2} & 0 & 0 \\
2 \xi_{2} & 2 \xi_{1}+2 \xi_{4}^{2} & -2 \xi_{4} & 4 \xi_{2} \xi_{4}-2 \xi_{3} \\
0 & -2 \xi_{4} & 2 & -2 \xi_{2} \\
0 & 4 \xi_{2} \xi_{4}-2 \xi_{3} & -2 \xi_{2} & 2 \xi_{2}^{2}
\end{array}\right)
$$

with the maximal rank in property (A1) being $k=3$. Moreover,

$$
\left.\operatorname{rank} D^{2} \psi\right|_{\xi_{2}=\xi_{3}=0}=2,\left.\operatorname{rank} D^{2} \psi\right|_{\xi_{1}=\xi_{2}=\xi_{3}=0}=1 .
$$

For $\xi_{2} \neq 0$ the kernel of the matrix $D^{2} \psi(\xi)$ is one dimensional:

$$
\operatorname{ker} D^{2} \psi(\xi)=\operatorname{span}\left\langle\left(\frac{\xi_{3}}{\xi_{2}}-\xi_{4}, 0, \xi_{2}, 1\right)\right\rangle \text {. }
$$

Therefore, the mapping $\varkappa$ of the direction of the line ker $D^{2} \psi$ corresponds to $\frac{\xi_{3}}{\xi_{2}}$ (after an analytic change of variables), and it has the essential singularities in the set

$$
\Omega^{\text {sing }}=\left\{\xi_{2}=\xi_{3}=0\right\} .
$$

Let us now consider other similar families of examples. Functions

$$
\psi\left(\xi_{1}, \xi_{2}, \xi_{3}, \xi_{4}\right)=\xi_{1} \xi_{2}^{k}+\left(\xi_{3}-\xi_{2} \xi_{4}\right)^{m}
$$

with $k, m \geq 2$ again lead to fibrations with essential singularities at $\xi_{2}=\xi_{3}=0$. The fibers are given by

$$
\operatorname{ker} D^{2} \psi(\xi)=\operatorname{span}\left\langle\left(\frac{m}{k} \frac{\left(\xi_{3}-\xi_{2} \xi_{4}\right)^{m-1}}{\xi_{2}^{k-1}}, 0, \xi_{2}, 1\right)\right\rangle,
$$

and so the set of essential singularities is also given by (2.2).

In $n$-dimensional space $\xi=\left(x_{1}, \ldots, x_{n-3}, y, z, w\right)$ define

$$
\psi(\xi)=y^{2} \sum_{i=1}^{n-3} x_{i}+(z-y w)^{2} .
$$


The level sets of the gradient $\nabla \psi$ have dimension $n-3$, and hence we have $k=3$. One can check that $\operatorname{dim} \Omega^{\text {sing }}=n-2$ (this also follows from dimension estimates of Theorem 2.1 with $k=3$ ).

Finally, consider

$$
\psi(x, y, z, v, w, s, t)=x y^{2}+s v^{2}+(z-y w-v t)^{2} .
$$

The maximal rank of the Hessian $D^{2} \psi$ equals $k=5$ and its kernel is spanned by the vectors

$$
\left(\frac{z-y w-v t}{y}, 0,0, y, 1,0,0\right),\left(0,0,0, v, 0, \frac{z-y w-v t}{v}, 1\right) .
$$

For $y=0$ and $z-v t=0$ the first vector has an essential singularity, while the second vector is continuous at $v \neq 0$. At the same time, for $v=0$ and $z-y w=0$, the second vector has an essential singularity while the first one is continuous for $y \neq 0$. Hence $\{y=0, z=v t\} \cup\{v=0, z=y w\} \subset \Omega^{\text {sing }}$, and thus $\operatorname{dim} \Omega^{\operatorname{sing}}=n-2$ (by the dimension estimates of Theorem 2.1). The intersection of spaces $\{y=0, z=v t\}$ and $\{v=0, z=y w\}$ is 4-dimensional.

In all the examples we observe that the set $\Omega^{\text {sing }}$ has an some affine structure: it is either affine itself, or it is a union of affine spaces. In fact, since the condition (A2) imposes a lot of affine structure for the whole problem, it may be the case that $\Omega^{\text {sing }}$ inherits these properties as well. So, we may formulate the following conjecture, as least in the case when $\Gamma=\nabla \psi$ for some holomorphic function $\psi$.

Conjecture. Let $\Omega$ be an open connected subset of $\mathbb{C}^{n}$ and let $\psi: \Omega \rightarrow \mathbb{C}$ be a holomorphic function. Suppose that the mapping $\Gamma=\nabla \psi$ satisfies properties (A1), (A2). Then the set $\Omega^{\text {sing }}$ of essential singularities of the corresponding fibration $\varkappa$ is a union of affine spaces.

We also note that these examples present singular fibrations for different dimensions of the fibers. On the other hand, the dimension of $\Omega^{\text {sing }}$ in all examples equals $n-2$. Under certain conditions it can be proved using dimension estimates in Theorem 2.1, or otherwise, that $\operatorname{dim}_{\xi} \Omega^{\text {sing }}=n-2$ at all (regular) points. However, our constructions are based on the same idea for the singularity. It would be interesting to investigate whether the condition $\operatorname{dim} \Omega^{\operatorname{sing}}=n-2$ is necessary for fibrations satisfying (2.1), or whether all affine spaces in the conjecture above would be $(n-2)$-dimensional.

\section{Relation to the symplectic geometry and Fourier integral operators}

The problem of the analysis of the set $\Omega^{\text {sing }}$ under conditions (A1) and (A2) is closely related to the symplectic geometry and to the theory of Fourier integral operators and the microlocal analysis in the following way.

Let $M$ be a smooth real analytic manifold. Let $T^{*} M$ denote the cotangent bundle of $M$, and let $\pi: T^{*} M \rightarrow M$ be the canonical projection. Let $\Lambda \subset T^{*} M$ be a conic analytic Lagrangian submanifold of $T^{*} M \backslash 0$ endowed with the standard symplectic form. Since we assume that all the spaces are analytic, we may always extend them to the complex domain, so it does not matter much whether we formulate the following relation in real or complex language.

Let $\Sigma$ be the regular part of $\pi(\Lambda)$, and we denote the conormal bundle of $\Sigma$ by

$$
N^{*} \Sigma=\left\{(m, \zeta) \in T^{*} M: m \in \Sigma, \zeta(\delta m)=0, \forall \delta m \in T_{m} \Sigma\right\} .
$$

Then the canonical projection $\pi$ defines the following restrictions:

$$
\begin{array}{ccccc}
N^{*} \Sigma & \subset & \Lambda & \subset T^{*} M \\
\downarrow & & \downarrow & & \downarrow \pi \\
\Sigma & \subset \pi(\Lambda) & & M
\end{array}
$$

It turns out that the generating function for the restriction $\pi: N^{*} \Sigma \rightarrow \Sigma$ leads to a mapping satisfying conditions (A1) and (A2). Let us explain this in more detail. 
By the Poincaré lemma of the symplectic geometry (see e.g. [1]), we can locally parameterise the Lagrangian manifold $\Lambda$ by some smooth analytic function $\phi$. This means that at a point $(m, \zeta) \in \Lambda$, we can locally write $\Lambda$ as

$$
\Lambda=\{(\nabla \phi(\zeta), \zeta)\}
$$

It turns out that the fibers of the mapping $\left.d \pi\right|_{\Lambda}$ correspond precisely to the level sets of ker $D^{2} \phi$, which are linear spaces in $\zeta$ in the conormal bundle (3.1). Thus, if we define

$$
\Gamma=\nabla \phi,
$$

then conditions (A1) and (A2) are satisfied for $\Gamma$. We can reduce the dimension further by using the fact that $\Lambda$ is conic, so we can factor out the conic variable from $\phi$, to obtain another function $\psi$, which would define $\Gamma$ by $(2.1)$.

Consequently, the set $\Omega^{\text {sing }}$ corresponds to the set of points where the fibration of $N^{*} \Sigma$ (as a union of affine spaces) is not continuously extendible to $\Lambda$. The details of this construction can be found in [7] or in $[8]$.

It was shown in [7] that the set $\Omega^{\operatorname{sing}} \cap \mathbb{R}^{n}$ is precisely the set where the so-called smooth factorization condition of Seeger, Sogge, and Stein [10] breaks down.

Let us briefly review now how the problem in terms of the symplectic geometry above is related to Fourier integral operators. Let $X$ and $Y$ be smooth paracompact real (analytic) manifolds of dimension $n$. Let $\Lambda^{\prime}$ be a conic (analytic) Lagrangian manifold in $\left(T^{*} X \backslash 0\right) \times\left(T^{*} Y \backslash 0\right)$, equipped with the symplectic form $\sigma_{X} \oplus-\sigma_{Y}$. Let $T \in I^{\mu}\left(X, Y ; \Lambda^{\prime}\right)$ be a Fourier integral operator of order $\mu$ with the canonical relation $\Lambda^{\prime}$, so that

$$
\Lambda=\left\{(x, \xi, y, \eta):(x, \xi, y,-\eta) \in \Lambda^{\prime}\right\} \subset T^{*}(X \times Y)
$$

is the wave front set of the integral kernel of the Fourier integral operator $T$. We consider non-degenerate Fourier integral operators $T$, for which the canonical relation $\Lambda^{\prime}$ locally is the graph of a symplectomorphism between $T^{*} X \backslash 0$ and $T^{*} Y \backslash 0$, equipped with canonical symplectic forms $\sigma_{X}$ and $\sigma_{Y}$. By the equivalence-of-phase-function theorem the set $\Lambda$ locally has the form

$$
\Lambda=\left\{\left(x, \nabla_{x} \Phi, y, \nabla_{y} \Phi\right): \nabla_{\theta} \Phi(x, y, \theta)=0\right\},
$$

for some phase function $\Phi=\Phi(x, y, \theta$, positively homogeneous of order one in $\theta$. With this phase, the Fourier integral operator $T$ can be written in the usual microlocal form

$$
T u(x)=\int_{Y} \int_{\mathbb{R}^{n}} e^{i \Phi(x, y, \theta)} a(x, y, \theta) u(y) d \theta d y,
$$

with some amplitude $a \in S_{1,0}^{\mu}$, a symbol of order $\mu$. Note, that the homogeneity of the canonical relation implies that rank $\left.d \pi_{X \times Y}\right|_{\Lambda} \leq 2 n-1$, where $\pi_{X \times Y}$ is the canonical projection from $T^{*}(X \times Y)$ to $X \times Y$. The result of [10] states that Fourier integral operators $T \in I^{\mu}\left(X, Y ; \Lambda^{\prime}\right)$ are locally continuous in $L^{p}$, provided $1<p<\infty$ and $\mu \leq-(n-1)|1 / p-1 / 2|$.

However, if the rank of $\left.d \pi_{X \times Y}\right|_{\Lambda}$ does not attain $2 n-1$, the order $\mu$ is not sharp and it depends on properties of the projection $\left.\pi_{X \times Y}\right|_{\Lambda}$. For example, it turns out that the regularity properties of Fourier integral operators in $L^{p}$ spaces with $p \neq 2$ depend on the maximal rank of the projection $\pi_{X \times Y}$ restricted to $\Lambda$. An important ingredient is the following smooth factorization condition for $\pi_{X \times Y}$. Suppose that there exists a number $k, 0 \leq k \leq n-1$, such that for every $\lambda_{0}=\left(x_{0}, \xi_{0}, y_{0}, \eta_{0}\right) \in \Lambda$, there exist a conic neighborhood $U_{\lambda_{0}} \subset \Lambda$ of $\lambda_{0}$ and a smooth homogeneous of zero order map $\pi_{\lambda_{0}}: \Lambda \cap U_{\lambda_{0}} \rightarrow \Lambda$ with constant rank, rank $d \pi_{\lambda_{0}}=n+k$, for which holds

$$
\pi_{X \times Y}=\pi_{X \times Y} \circ \pi_{\lambda_{0}} .
$$

Denoting $M=X \times Y$, we obtain that condition (3.3) is equivalent to the condition that the fibration of $N^{*}(\Sigma)$ by affine fibres is smoothly extendible to $\Lambda$ (modulo a correction for zero sections). Here $\pi=\pi_{X \times Y}$, and $\Sigma=\pi\left(N^{*}(\Sigma)\right)$ is the regular part of $\pi(\Lambda)$, which is the singular support of the integral kernel of operator $T$ in $(3.2)$. 
Acknowledgements. The author was supported by the EPSRC Leadership Fellowship.

\section{References}

[1] J. J. Duistermaat, Fourier integral operators. Birkhäuser, Boston, 1996.

[2] S. Łojasiewicz. Introduction to complex analytic geometry. Birkhäuser, Basel, 1991.

[3] F. Nicola. Boundedness of Fourier integral operators on Fourier Lebesgue spaces and affine fibrations. Studia Math., 198 (2010), 207-219.

[4] R. Remmert. Holomorphe und meromorphe Abbildungen komplexer Räume. Math. Ann. 133 (1957), 328-370.

[5] M. Ruzhansky. Analytic Fourier integral operators, Monge-Ampere equation and holomorphic factorization. Arch. Math., 72 (1999), 68-76.

[6] M. Ruzhansky. On singularities of affine fibrations of certain types. Russian Math. Surveys, 55(2000), 353-354.

[7] M. Ruzhansky. Singularities of affine fibrations in the regularity theory of Fourier integral operators. Russian Math. Surveys, 55 (2000), 93-161.

[8] M. Ruzhansky. Regularity theory of Fourier integral operators with complex phases and singularities of affine fibrations. CWI Tracts, vol. 131, 2001.

[9] M. Ruzhansky. On the failure of the factorization condition for non-degenerate Fourier integral operators. Proc. Amer. Math. Soc., 130 (2002), 1371-1376.

[10] A. Seeger, C. D. Sogge, E. .M. Stein. Regularity properties of Fourier integral operators. Ann. of Math.134 (1991), $231-251$. 